\title{
LOS MULTIOPPIINAE DEL SUR DE ESPAÑA II. EL GÉNERO RAMUSELLA CON DESCRIPCIÓN DE UNA NUEVA ESPECIE (ACARI, ORIBATIDA, OPPIIDAE)*
}

\author{
A. Arillo (**) y L.S. Subías (**)
}

\section{RESUMEN}

En el presente trabajo se estudia la distribución de las especies del género Ramusella Hammer, 1962 en el sur de España. Se describe la nueva especie Ramusella (Rectoppia) eduardoi sp. nov.

Palabras clave: Acaros, Oribátidos, Oppiidae, Multioppiinae, Ramusella, España

\begin{abstract}
Multioppiinae from the South of Spain II. Genus Ramusella with description of a new species (Acari, Oribatida, Oppiidae)

In this paper species belonging to the genus Ramusella Hammer, 1962 from the South of Spain are studied. A new species, Ramusella (Rectoppia) eduardoi sp. nov. is described.
\end{abstract}

Key words: Oribatid mites, Oppidae, Multioppiinae, Ramusella, Spain

\section{Introducción}

Esta segunda parte del estudio sobre la subfamilia Multiopiinae Balogh, 1983 se dedicará al género Ramusella Hammer, 1962 del que se han obtenido gran cantidad de ejemplares pertenecientes a 14 especies y tres subespecies distintas.

El género Ramusella es tal vez el mejor representado en la fauna ibérica. Se trata de un género de sistemática complicada, a pesar de que Subías (1980a) lo simplificó dividiéndolo en tres subgéneros que posteriormente se han ampliado hasta cinco.
Un problema frecuente en este género es la antigüedad de algunas descripciones (que por añadidura suelen ser bastante deficientes) lo que produce que la identidad de algunas especies sea confusa y, consecuentemente, aparezcan abundantes casos de sinonimias.

\section{Material}

El material, depositado en la Colección de la Cátedra de Entomología del Departamento de Biología Animal I de la Facultad de Biología de la Universidad Complutense de Madrid, se encuentra

\footnotetext{
* Trabajo financiado por la DGICYT (Proyecto Fauna Ibérica III, $\mathrm{n}^{\circ}$ PB92-0121)

** Dpto. Biología Animal I (Entomología). Facultad de Biología. Universidad Complutense. 28040 Madrid.
} 
conservado, parte en preparaciones semipermanentes con medio de Hoyer y parte en ácido láctico al $70 \%$.

El material procede de las siguientes muestras:

HUELVA 1A: El Campillo; U.T.M. 29SQB37; suelo de pinar; 7-IV-1977; L.S. Subías leg.

HUELVA 2A: La Palma del Condado; U.T.M. 29SQB14; hojarasca de Cistus salvifolia; 7-IV-1977; R. Outerelo leg.

HUELVA 3A: Galaroza; U.T.M. 29SQC00; musgo y tierra de base de tronco de castaño; 8-IV-1977; L.S. Subías leg.

HUELVA 3B: Galaroza; U.T.M. 29SQC00; suelo de castañar; 8-IV-1977; R. Outerelo leg.

HUELVA 4A: Cumbres de En Medio; U.T.M. 29SPC91; hojarasca de encinar con jara y lavándula; 28-IV-1983; L.S. Subías y M.A. Ferrández leg.

HUELVA 5A: Coto de Doñana; U.T.M. 29SQA29; hojarasca y césped de pinar piñonero; 1-X-1986; L.S. Subías leg.

HUELVA 6A: Santa Olalla de Cala; U.T.M. 30SQB49; musgos ripícolas; 30-X-1986; $\mathrm{R}$. Outerelo leg.

SEVILLA 7A: Cazalla de la Sierra; U.T.M. 30STH50; suelo bajo piedra en castañar; 25-III-1978; R. Outerelo leg.

SEVILLA 7B: Cazalla de la Sierra ; U.T.M. 30STH50; suelo de castañar; 25-III-1978; R. Outerelo leg.

SEVILLA 7C: Cazalla de la Sierra ; U.T.M. 30STH50; hojarasca de alcornoque; 25-III-1978; R. Outerelo leg.

SEVILLA 8A: Cuesta de la Media Fanega, N-630; U.T.M. 29SQB47; hojarasca de encina; 25-II-1974; S. Pérez leg.

SEVILLA 8B: Cuesta de la Media Fanega, N-630, U.T.M. 29SQB47; hojarasca y suelo de encina; 5-III-1983; L.S. Subías, M.A.Ferrández y E.Ruiz leg.

CÁDIZ 9A: Los Barrios; U.T.M. 30STF70; hojarasca de Quercus canariensis; 24-II-1974; S. Pérez leg.

CÁDIZ 9B: Los Barrios; U.T.M. 30STF70; islote arenoso de Juncus en el río; 24-II-1974; L.S. Subías leg.

CÁDIZ 9C: Los Barrios; U.T.M. 30STF70; hojarasca de Quercus canariensis; 24-II-1974; L.S. Subías leg.

CÁDIZ 10A: Prado del Rey; U.T.M. 30STF77; hojarasca de olivo y roble; 2-IV-1983; E. Ruiz leg.

CÁDIZ 11A: Entre Ubrique y Puerto Galis; U.T.M. 30STF75; hojarasca de alcornoque; 5-III-1981; M.A. Ferrández leg.

CÓRDOBA 13A: Alcolea; U.T.M. 30SUH50; hojarasca de olivo; 22-II-1974; S. Pérez leg.

CÓRDOBA 14A: Villanueva del Duque; U.T.M. 30SUH14; hojarasca y suelo de encinar con jaras; 29-IV-1983; L.S. Subías y M.A. Ferrández leg.

CÓRDOBA 15A: Santa Eufemia; U.T.M. 30SUH37; hojarasca de encinar con algunas jaras; 1-XII-1983; L.S. Subías, M.A. Ferrández y E. Ruiz leg.

CÓRDOBA 16A: El Tejar; U.T.M. 30SUG62; suelo de pinar repoblado con retamas y musgos; 22-III-1983; L.S. Subías M.A. Ferrández y E. Ruiz leg.

CÓRDOBA 17A: Venta del Charco; U.T.M. 30SUH82; musgo y hojarasca de encinar; 1-XII-1983; L.S. Subías, M.A. Ferrández y E. Ruiz leg.

CÓRDOBA 18A: Rasos; U.T.M. 30SUH81; hojarasca de encina y jara en zona montañosa; 1-XII-1983; L.S. Subías, M.A. Ferrández y E. Ruiz leg.

CÓRDOBA 19A: Posadas, La Plata; U.T.M. 30SUG18; tierra de talud en un arroyo temporal; 29-XI-1986; J.P. Zaballos leg.
CÓRDOBA 19B: Posadas, La Plata; U.T.M. 30SUG18; tierra de talud en un arroyo temporal; 25-V-1991; J.P. Zaballos leg.

CÓRDOBA 20A: Pedro Abad; U.T.M. 30SUH70; base de tronco de olivo; 22-X-1989; M.D. Martínez leg.

CÓRDOBA 21A: El Carpio; U.T.M. 30SUH 60; hojarasca de chopo junto al Guadalquivir; 29-X-1989; L.S. Subías leg.

MÁLAGA 22B: Ronda de Málaga (Coto de la Nava); U.T.M. 30SUF06; musgo y hojarasca de pinsapo; 23-II-1974; S. Pérez leg.

MÁLAGA 23A: Sierra de Ronda; U.T.M. 30SUF16; hojarasca y tierra al pie de un tocón de pinsapo; 23-II-1974; R. Outerelo leg.

MÁLAGA 24A: Los Reales; U.T.M. 30SUF03; hojarasca de pinsapo bajo musgo; 23-II-1974; R. Outerelo y L.S. Subías leg.

MÁLAGA 25A: Alora; U.T.M. 30SUF48; suelo de cultivo de olivo; 22-III-1983; L.S. Subías, M.A. Ferrández y E. Ruiz leg.

MÁLAGA 26A: Puerto de Marbella, Sierra Blanca, Ojén; U.T.M. 30SUF34; suelo en pinar con enebro; 21-IX-1985; M.A. Ferrández y C.M. Veiga leg.

JAÉN 28A: Sierra de Cazorla; U.T.M. 30SWH00; hojarasca de chopo y de olmo a $20 \mathrm{~cm}$ de profundidad; 3-VII-1978; R. Outerelo leg.

JAÉN 28B: Sierra de Cazorla; U.T.M. 30SWH00; hojarasca de chopo y olmo a $20 \mathrm{~cm}$ de profundidad; 3-VII-1978; R. Outerelo leg.

JAÉN 28C: Sierra de Cazorla; U.T.M. 30SWH00; hojarasca de chopo y olmo; 3-VII-1978; R. Outerelo leg.

JAÉN 30B: Despeñaperros; U.T.M. 30SVH54; hojarasca de encina; 22-II-1974; R. Outerelo leg.

JAÉN 31A: Mancha Real (Sierra Majina); U.T.M. 30SVG48; encinar en suelo calizo; 6-IV-1980; M.A. Ferrández leg.

GRANADA 33A: Portugos, Fuente Agrilla; U.T.M. 30SVF79; hojarasca de castaño; 30-III-1983; R. Outerelo leg.

GRANADA 34A: Orjiva (Río Chico); U.T.M. 30SVF68; hojarasca de sauce, chopo y zarza; 30-III-1983; R. Outerelo leg.

GRANADA 35A: Capileira; U.T.M. 30SVF69; hojarasca de roble; 2-IX-1978; J. Berzosa leg.

ALMERÍA 39A: Tabernas; U.T.M. 30SWG50; hojarasca y tierra de Tamarix y Juncus; 22-XI-1975; L.S. Subías leg.

ALMERÍA 40A: Tahal, Sierra de Filabres; U.T.M. 30SWG62; hojarasca de Pinus halepensis; 22-XI-1975; R. Outerelo y L.S. Subías leg.

ALMERÍA 41A: Rodalquilar; U.T.M. 30SWF87; tierra a pie de palmito; 23-XI-1975; R. Outerelo y L.S. Subías leg.

ALMERÍA 42B: Macael, Sierra de Filabres; U.T.M. 30SWG63; algo de hojarasca, musgo y tierra de protosuelo; 22-XI1975; L.S. Subías leg.

ALMERÍA 42C: Macael, Sierra de Filabres; U.T.M. 30SWG63; hojarasca variada de pinar con denso sotobosque; 22-XI1975; L.S. Subías leg.

ALMERÍA 43A: Cabo de Gata; U.T.M. 30SWF76; arena entre retamas; 23-XI-1975; R. Outerelo y L.S. Subías leg.

ALMERÍA 44A: El Marchal; U.T.M. 30SWF38; suelo en encinar con retama y genista; 24-III-1983; L.S. Subías, M.A. Ferrández y E. Ruiz leg.

MURCIA 45A: Sierra de Espuña, a 1500 m; U.T.M. 30SXG29; hojarasca de Juniperus phoenicea; 30-IX-1981; J.J. Presa leg. 
MURCIA 45B: Sierra de Espuña; U.T.M. 30SXG29; hojarasca diversa de pino, coscoja y pistacea; 31-X-1980; J.J. Presa y M.D. García leg.

MURCIA 46A: Albudeite, a 200 m; U.T.M. 30SXH40; hojarasca de Brachypodium ramsum; 5-V-1978; R. Outerelo leg.

MURCIA 51A: Sierra Cresta del Gallo; U.T.M. 30SXH60 ; hojarasca de romero sobre calizas; 31-X-1977; R. Outerelo leg.

MURCIA 53A: Portman, Peña del Águila; U.T.M. 30SXG96; hojarasca y suelo calizo y seco de pinar mezclado con Tetraclinis articulata, esparto, palmito, romero, pistacea, tomillo y lavándula; 27-I-1983; L.S. Subías y E. Ruiz leg.

\section{Resultados}

Subgénero Ramusella (Ramusella) Hammer, 1962

Ramusella (Ramusella) clavipectinata s. str. (Michael, 1885)

Notaspis clavipectinata Michael, 1885

Material estudiado: Huelva 2A (155 ej.); Huelva 3A (3 ej.); Huelva 4A (461 ej); Huelva 6A (7 ej.); Sevilla 7A (464 ej.); Sevilla 7B (96 ej.); Sevilla 7C (115 ej.); Sevilla 8A (85 ej.); Sevilla 8B (199 ej.); Cádiz 9A (1 ej.); Cádiz 9B (811 ej.); Córdoba 13A (76 ej.); Córdoba 14A (77 ej.); Córdoba 17A (124 ej.); Córdoba 21A (4 ej.); Jaén 28A (71 ej.); Jaén 28B (62 ej.); Jaén 28C (27 ej.); Jaén 31A (14 ej.); Granada 34A (6 ej.); Almería 43A (1 ej.); Almería 44A (22 ej.); Murcia 45B (1 ej.).

Dimensiones: $250-360 \times 138-200 \mu \mathrm{m}$.

Discusión: Esta especie ha sido habitualmente citada en España como Ramusella (R.) assimilis, siendo considerado el epíteto "clavipectinata" como asignable a Ramusella (Insculptoppia) (Subías, 1980a). Sin embargo, tras la revisión que Beck \& Woas (1991) realizan sobre el tipo de Notaspis clavipectinata Michael, 1885 es evidente que se trata de la misma especie, por lo que podemos confirmar la sinonimia que con dudas realizan Subías \& P. Balogh (1989).

Patas (Fig. 1): Hemos seleccionado esta especie para estudiar el modelo quetotáxico de las patas del género debido a su abundancia. Es una especie que no ha sufrido ninguna reducción y sus caracteres quetotáxicos son triviales, aunque, dado el gran número de ejemplares estudiados, hemos encontrado cierta variabilidad, apareciendo

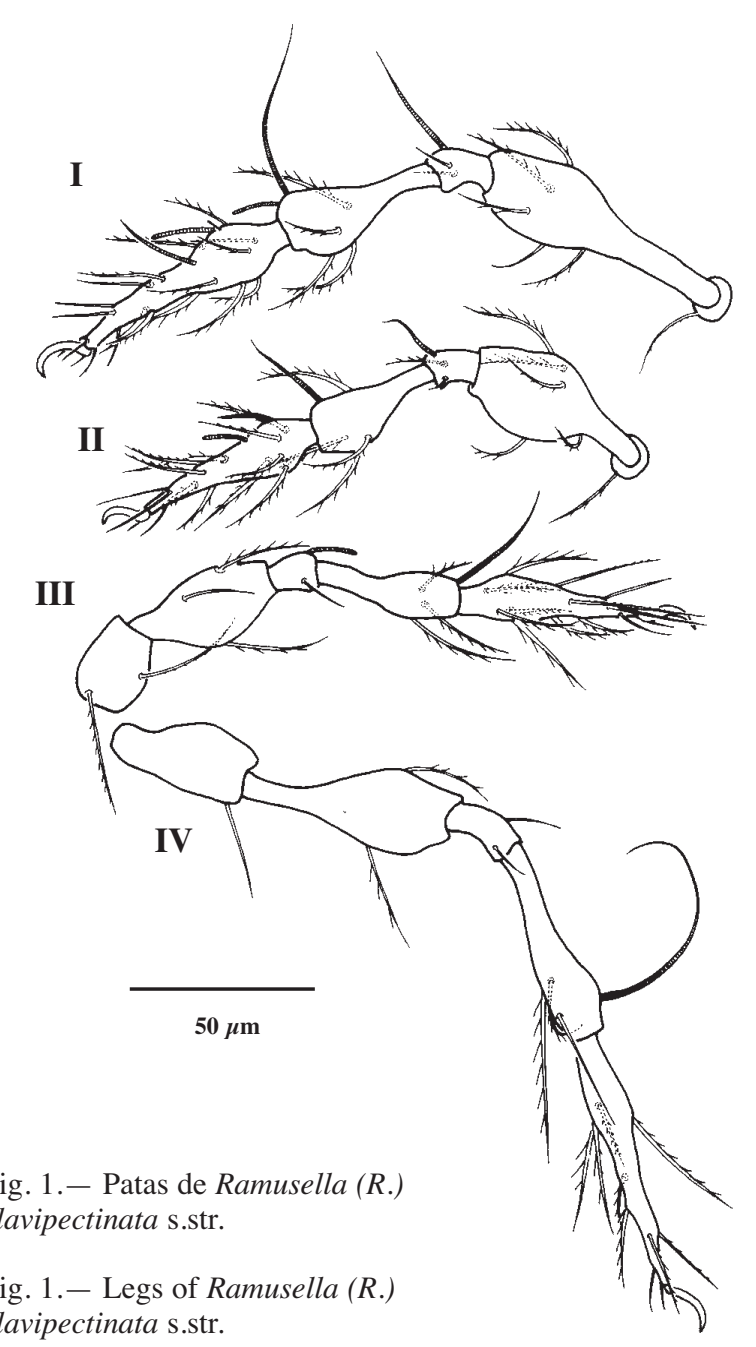

clavipectinata s.str.

en un ejemplar la pérdida de un solenidio del tarso II y en otro ejemplar la pérdida de una seta ventral del tarso III.

Distribución: Es una especie posiblemente holártica que aparece frecuentemente en España, habiendo sido citada de Ávila, Huesca, Madrid, Navarra, Salamanca, Segovia, Toledo y Vizcaya. También ha sido citada en el Algarve, Menorca, Tenerife y Madeira. En nuestra zona de estudio era conocida de Jaén (Subías, 1980b) citada como Oppia insculpta.

Biología: Subías (1980a) y Subías \& Rodríguez (1987) apuntan que se trata de una especie con preferencia por zonas húmedas con abundante materia orgánica, aunque debe tener una valencia ecológica más amplia puesto que en nuestro estudio aparece también en muestras pobres en materia orgánica y medios xéricos. 


\section{Ramusella (Ramusella) clavipectinata assimiloides stat. nov. Subías y Rodríguez, 1987 \\ Ramusella (R.) assimiloides Subías y Rodríguez, 1987}

Material estudiado: Huelva 5A (148 ej.); Cádiz 10A (26 ej.); Cádiz 11A (96 ej.); Córdoba 15A (318 ej.); Córdoba 16A (130 ej.); Córdoba 18A (140 ej.); Málaga 25A (21 ej.); Jaén 30B (62 ej.); Almería 40A (1 ej.); Almería 41 A (9 ej.); Almería 42C (20 ej.); Murcia 46A (1 ej.); Murcia 51A (5 ej.); Murcia 53A (16 ej.).

Dimensiones: 244-350 $\mu \mathrm{m} \times 125-200 \mu \mathrm{m}$.

Discusión: De los tres caracteres que Subías \& Rodríguez (op. cit.) utilizan para diferenciar $R .(R$.) assimilis de $R$.(R.) assimiloides, forma de la prominencia rostral, ramas del sensilo y orientación del discidio, parece que sólo el aspecto de las ramas del sensilo es constante, ya que los otros caracteres son bastante variables aún dentro de una población. Pensamos pues que la forma de las ramas del sensilo es el único caracter válido para separar las dos formas, pero, a la vez, creemos que dentro de una especie tan variable como parece ser $R$.(R.) clavipectinata esta diferencia podría entrar dentro de la variabilidad intraespecífica, por lo que hemos optado por considerar $R$.(R.) assimiloides como una subespecie de $R$.(R.) clavipectinata siendo posible que incluso se pudieran considerar como simples morfotipos y, en el futuro, realizar la sinonimia de ambas especies.

Distribución: Conocida solamente en España, concretamente de las provincias de Álava, Albacete, Burgos, Ciudad Real, Madrid, Soria, Teruel y Vizcaya. En nuestra zona de estudio estaba citada en la provincia de Murcia (Subías \& Rodríguez, op. cit.).

Biología: En su descripción original fue hallada en distintos medios dentro de sabinares albares. También en nuestras muestras parece confirmarse la amplia valencia ecológica de esta subespecie.

\section{Ramusella (Ramusella) junonis C.Pérez-Íñigo, 1986}

Material estudiado: Huelva 3A (1 ej.); Huelva 3B (12 ej.); Huelva 4A (294 ej.); Sevilla 7A (12 ej.); Sevilla 7C (18 ej.); Córdoba 15A (2 ej.).
Dimensiones: $240-312 \times 125-187 \mu \mathrm{m}$.

Discusión: Existen pequeñas diferencias con los ejemplares utilizados en la descripción original. Nuestros ejemplares pueden presentar protuberancia rostral, las setas interlamelares son más gruesas, pueden tener ramas internas en el sensilo y las setas notogastrales son ciliadas. En todo caso estas características han aparecido aisladas también en nuestros ejemplares por lo que pensamos que se trata de variabilidad intraespecífica que puede ser bastante acusada en las especies de este género.

Distribución: Sólo era conocida hasta ahora de la isla de La Gomera, por lo que se trata de la primera cita continental.

Biología: En nuestras muestras ha aparecido asociada a encinas, castaños y alcornoques.

\section{Ramusella (Ramusella) puertomonttensis} Hammer, 1962

Material estudiado: Córdoba 16A ( 1 ej.); Jaén 31A (184 ej.); Granada 33A (5 ej.); Granada 34A (516 ej.).

Dimensiones: $243-288 \times 137-163 \mu \mathrm{m}$.

Discusión: La presencia de algunos individuos con seta $c_{2}$ desarrollada, incluso sólo en una mitad del cuerpo, parece indicar que la especie que Subías (1980a) crea para los ejemplares con seta $c_{2}$ (denominándolos Ramusella (R.) tasetata), pueda tratarse de una sinonimia. Por su parte los ejemplares estudiados por Iturrondobeitia \& Saloña (1988) y determinados como Ramusella (R.) cf. puertomonttensis, parecen tratarse de dos especies (que ellos denominan forma a y b) apareciendo la seta $c_{2}$ desarrollada en la forma "b" que es la más semejante a $R .(R$.) puertomonttensis. Es posible que los denominados forma "a" puedan incluso corresponder a una Ramusella (Insculptoppia) como veremos más adelante (Saloña, comunicación personal).

Distribución: especie aparentemente cosmopolita, ha aparecido en España en las provincias de Álava, Burgos, Madrid, Toledo y Vizcaya. También es conocida de Madeira, Tenerife y La Gomera. 
Biología: Subías (1980a) la considera asociada a medios con cierto grado de humedad, aunque en nuestras muestras ha aparecido también en medios más bien secos.

\section{Ramusella (Ramusella) sengbuschi Hammer, 1968}

Material estudiado: Granada 33A (8 ej.); Granada 35A (86 ej.); Granada 38A (29 ej.).

Dimensiones: $247-290 \times 125-160 \mu \mathrm{m}$.

Discusión: Como ya pusieron de manifiesto Fujita \& Fujikawa (1986) y Arillo et al. (1988) se trata de una especie sumamente variable, lo que parece ser una característica común en el género.

Distribución: Conocida de Nueva Zelanda, Japón, Java, Filipinas y España, habiendo sido encontrada hasta ahora en las provincias de Madrid y Toledo.

Biología: Suele aparecer en medios alterados por el hombre aunque en nuestras muestras no es así, sino que aparece en hojarasca de diversas especies.

Subgénero Ramusella (Insculptoppia) Subías, 1980

\section{Ramusella (Insculptoppia) anuncata}

Subías y Rodríguez, 1986

Material estudiado: Huelva 3A (1 ej.).

Dimensiones: $282 \times 138 \mu \mathrm{m}$.

Discusión: Las diferencias observadas respecto a la descripción original no parecen ser debidas más que a una probable variabilidad intraespecífica como ya hemos visto en otras especies del género.

Distribución: Especie sólo hallada en España, concretamente en las provincias de Madrid, Toledo y Valencia.

Biología: Especie posiblemente xerófila, con preferencia por suelos minerales o alterados por el hombre. En nuestro estudio en cambio ha aparecido en una muestra de musgo y tierra en la base de un castaño.

\section{Ramusella (Insculptoppia) berninii \\ (Pérez-Íñigo, 1975) \\ Oppia bernini Pérez-Íñigo, 1975}

Material estudiado: Huelva 1A (1 ej.).

Dimensiones: 350 x $188 \mu \mathrm{m}$.

Distribución: Especie conocida de Italia y España, concretamente de las provincias de Guadalajara y Madrid.

Biología: Especie descrita en una cueva de Guadalajara fue posteriormente encontrada en diversas cuevas italianas. Pero también había sido encontrada en medios no cavernícolas, concretamente en madera descompuesta. En nuestro estudio ha aparecido en una muestra de suelo de pinar.

\section{Ramusella (Insculptoppia) elliptica (Berlese, 1908) \\ Lohmannia elliptica Berlese, 1908}

Material estudiado: Sevilla 7A (1 ej.); Córdoba 19A (1 ej.); Málaga 26A (1 ej.).

Dimensiones: 237-250 x 123-127 $\mu \mathrm{m}$.

Distribución: Considerada como distribuida en el Paleártico meridional, es frecuente en España, donde se conoce en las provincias de Álava, Burgos, Madrid, Navarra, Segovia y Vizcaya, aunque es muy probable que gran cantidad de citas de Oppia insculpta y Oppia assimilis se refieran a esta especie. También ha sido citada de Tenerife y Madeira.

Biología: Especie de amplia valencia ecológica en nuestro estudio ha aparecido bajo piedra en un castañar, en el talud de un arroyo y en el suelo de un pinar con enebros.

\section{Ramusella (Insculptoppia) insculpta (Paoli, 1908) \\ Dameosoma insculptum Paoli, 1908}

Material estudiado: Jaén 28A (44 ej.); Jaén 28B (2 ej.); Jaén $28 \mathrm{C}(2 \mathrm{ej}$.

Dimensiones: 231-250 x 113-138 $\mu \mathrm{m}$. 
Discusión: Nuestros ejemplares presentan algunas peculiaridades en el prodorso. Éste tiene el rostro entero. Las setas rostrales son largas y arqueadas aunque en ciertas orientaciones da la sensación de que pudieran tener un cierto acodamiento. Estas setas están barbuladas en su lado externo, pero en la mitad distal apenas hay bárbulas. Los alveolos de inserción están a una distancia intermedia, ni tan separados como es típico de Ramusella (Insculptoppia) ni tan próximos como en Ramusella (Ramusella). Las setas lamelares e interlamelares son más cortas que las rostrales y están ligeramente barbuladas. El sensilo presenta un tallo largo, con una cabeza apenas ensanchada en cuyo lado externo porta una hilera de unas diez ramas que en su tramo central son muy largas.

En el notogáster destacan las setas $c_{2}$ que están desarrolladas aunque son diminutas. Fisuras ia e im patentes.

La especie es complicada por la mezcla de caracteres de sus setas rostrales. A la especie que más se asemeja es a Ramusella (R.) puertomonttensis pero en esta especie las setas rostrales son indiscutiblemente acodadas. Por otra parte Saloña (comunicación personal) quien ha estudiado el holotipo depositado en Florencia, opina que los ejemplares del sur de España no pertenecen a la verdadera Ramusella (I.) insculpta que sería notablemente mayor y cuyo sensilo tendría las ramas más cortas, caracteres estos con los que encajan los ejemplares considerados como Ramusella (R.) puertomonttensis forma "a" por Iturrondobeitia \& Saloña (op . cit.) del País Vasco que, además, no tienen la seta $c_{2}$ desarrollada. Por consiguiente, en tanto sea acalarada la verdadera identidad de estos ejemplares los incluimos, con muchas reservas, dentro de Ramusella (Insculptoppia) insculpta .

Distribución: esta especie se considera distribuida por el paleártico sur. En España ha sido frecuentemente citada, pero, como se ha comprobado en ocasiones, ha sido confundida con otras especies del género Ramusella, por lo que su distribución es desconocida, aunque al menos deben pertenecer a esta especie los ejemplares de Álava, Burgos y Vizcaya estudiados por Iturrondobeitia \& Saloña (op. cit.) y los procedentes de Isla Grosa (Murcia) (Subías, 1980a).
Biología: Especie que, posiblemente, tenga una valencia ecológica amplia. En nuestro estudio ha aparecido en varias muestras de hojarasca de chopo y olmo.

\section{Ramusella (Insculptoppia) terricola Subías y Rodríguez, 1986}

Material estudiado: Sevilla 7A (13 ej.); Sevilla 7B (77 ej.); Sevilla 7C (3 ej.); Sevilla 8B (1 ej.).

Dimensiones: $194-225$ x 94-113 $\mu \mathrm{m}$.

Distribución: Especie conocida de España, concretamente de las provincias de Madrid, Palencia, Soria, Teruel y Valencia. Asimismo es conocida, en nuestra área de estudio de Murcia.

Biología: Recolectada habitualente en suelos minerales, en nuestro muestreo fue recogida en varios medios en castañar y encinar.

Subgénero Ramusella (Rectoppia) Subías, 1980

Ramusella (Rectoppia) eduardoi sp. nov. (Figura 2)

Material estudiado: Huelva 2A (22 ej.); Cádiz 9A (3 ej.); Cádiz 9C (2 ej.); Cádiz 10A (8 ej.); Almería 40A (1 ej.); Almería 42B (1 ej.); Murcia 51A (6 ej.).

Dimensiones y tegumento: Los ejemplares estudiados tienen unas dimensiones comprendidas entre 219-247 $\mu \mathrm{m}$ de longitud por 106-125 $\mu \mathrm{m}$ de anchura. La coloración es clara debido a la escasa esclerotización.

Prodorso (Fig. 2A y 2B): Rostro proyectado anteriormente $\mathrm{y}$ hacia abajo formando una estructura rectangular simétrica respecto al plano sagital, aunque en algunos ejemplares está más desarrollado un lado que el otro resultando asimétricos respecto a dicho plano. La setas rostrales son cortas, divergentes y ciliadas en su lado externo. Las setas lamelares tienen un tamaño similar pero son más finas y lisas. Las setas interlamelares son muy cortas y lisas. El sensilo tiene un tallo bien desarrollado con una maza diferen- 


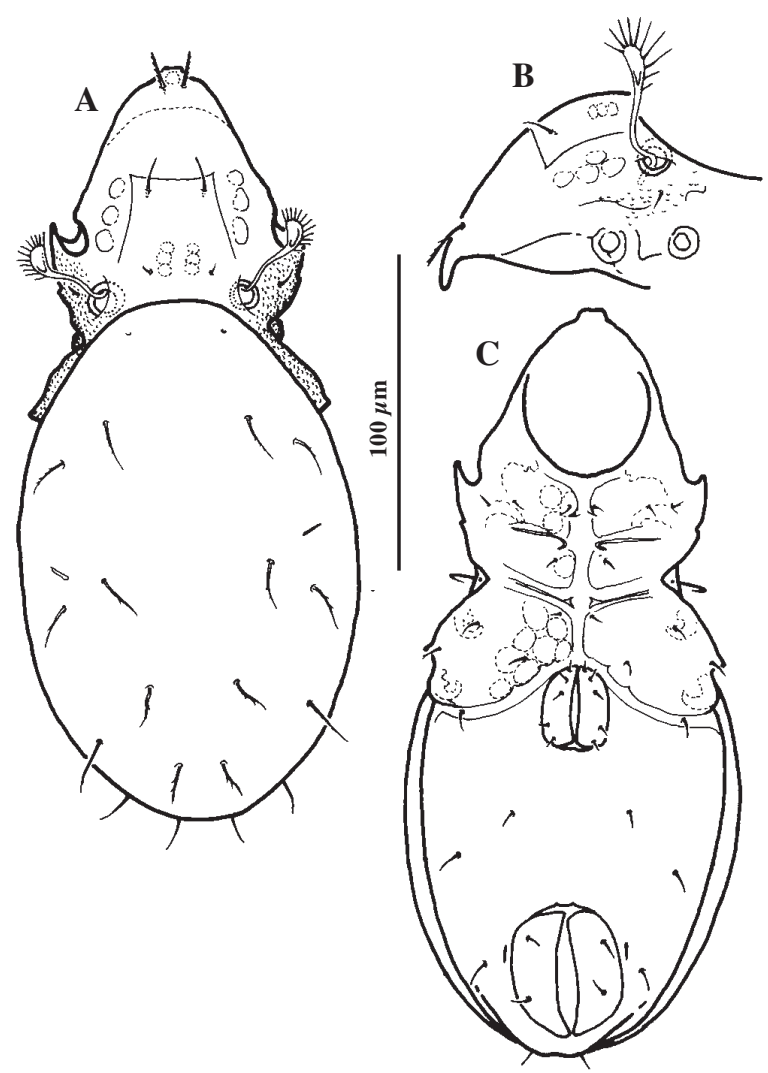

Fig 2.- Ramusella (Rectoppia) eduardoi. a) Visión dorsal. b) Visión ventral. c) Visión lateral del prodorso.

Fig 2.- Ramusella (Rectoppia) eduardoi. a) Dorsal view. b) Ventral view. c) Lateral view of the prodorsum.

ciada y redondeada que porta en su lado externo una hilera de unas 12 ramas cortas y similares. La seta exobotrídica es muy corta y lisa. Las líneas lamelares y translamelar están bien desarrolladas. Aparecen los tres pares de áreas claras prodorsales habituales y un denso granulado en la zona exobotrídica.

Notogáster (Fig. 2A): Nueve pares de setas notogastrales cortas y portando entre una y tres bárbulas. Las setas $c_{2}$ son diminutas o faltan del todo. Las fisuras im están bien desarrolladas.

Región ventral (Fig. 2C): Fórmula quetotáxica habitual (3-1-3-3) siendo todas las setas cortas y lisas (salvo las $3_{\mathrm{c}}$ algo más largas). Aparecen los habituales cinco pares de setas genitales, un par de adgenitales, dos pares de anales y tres pares de adanales todas cortas y lisas. El par $a d_{1}$ es postanal.
Las fisuras iad son paraanales. El discidio es aguzado pero escasamente recurvado.

Discusión: Especie próxima por su tamaño y forma del sensilo a R.(Rectoppia) mihelcici y a $R .($ Rectoppia) rhinina, presentando, de hecho, caracteres intermedios entre ambas, referidos fundamentalmente a la forma del rostro que en $R .(R$.) mihelcici es entero o, en todo caso, ligeramente apuntado y en $R$.(R.) rhinina desarrolla una prolongación (mayor que en la nueva especie) que en sus ángulos externos incluso llega a ser trilobulado.

Biología: Ha aparecido en varias muestras de hojarasca pero de muy diferentes especies, por lo que parece que su valencia debe ser alta.

Derivatio nominis: Hemos dedicado esta especie al botánico Dr. Eduardo Barrón López.

\section{Ramusella (Rectoppia) fasciata s. str. (Paoli, 1908) \\ Dameosoma fasciatum Paoli, 1908}

Material estudiado: Almería 39A (46 ej.).

Dimensiones: $300-325 \times 156-175 \mu \mathrm{m}$

Discusión: Esta subespecie ha sido frecuentemente confundida con la otra subespecie $R$.(R.) fasciata sahariensis pero la forma del sensilo (con las ramas centrales mucho más largas en $R$.(R.) fasciata s.str.) y la longitud de las setas notogastrales (mucho más largas en $R$.(R.) fasciata sahariensis) las separa con facilidad.

Distribución: Considerada por Subías \& P. Balogh (op. cit.) como holártica, ha sido citada en diversas ocasiones en España si bien, como hemos comprobado después, la mayoría de las citas eran de $R$.(R.) fasciata sahariensis siendo esta la confirmación de la verdadera presencia de esta subespecie en España.

Biología: La biología de esta subespecie es aún bastante desconocida por sus problemas taxonómicos. En nuestro estudio ha aparecido en una muestra de hojarasca y tierra de Tamarix y Juncus. 


\section{Ramusella (Rectoppia) fasciata sahariensis (Hammer,1975) \\ Oppia sahariensis Hammer, 1975}

Material estudiado: Córdoba 20A (4 ej.); Jaén 28A (9 ej.); Jaén 28C (4 ej.); Granada 33A (1 ej.)

Dimensiones: 314-362 x 163-200 $\mu \mathrm{m}$.

Distribución: Subespecie mediterránea que era conocida (con seguridad) en España de la provincia de Albacete y en nuestra área de estudio de Jaén.

Biología: Especie que suele aparecer en medios con abundante materia orgánica. En nuestro estudio ha sido recolectada en hojarasca de castaño, olmo y chopo y en la base de un tronco de olivo.

\section{Ramusella (Rectoppia) mihelcici (Pérez-Íñigo, 1965) \\ Oppia mihelcici Pérez-Íñigo, 1965}

Material estudiado: Cádiz 9B (1 ej.); Cádiz 11A (1 ej.); Córdoba 19A (1 ej.); Córdoba 19B (3 ej.); Jaén 28A (105 ej.); Jaén 28B (11 ej.); Jaén 28C (4 ej.); Jaén 30B (25 ej.); Jaén 31A (35 ej.); Almería 43A (1 ej.); Murcia 45A (1 ej.); Murcia 45B (1 ej.).

Dimensiones: 203-251 x 113-126 $\mu \mathrm{m}$.

Distribución: Especie distribuida en el Paleártico sur ha sido frecuentemente citada en España, concretamente en las provincias de Álava, Albacete, Cuenca, Huesca, León, Madrid, Palencia, Segovia, Teruel, Valencia, Vizcaya y Zaragoza. También se conoce de Menorca y Azores. En nuestra área de estudio se conocía en las provincias de Jaén y Murcia.

Biología: Especie típicamente euedáfica y xerófila. En nuestro estudio aparece asociada a medios arenosos pero también ha aparecido ligada a hojarasca de muy diversas especies.

\section{Ramusella (Rectoppia) rhinina} Subías y Mínguez, 1981

Material estudiado: Huelva 4A (2 ej.); Córdoba 14A (5 ej.).

$$
\text { Dimensiones: } 244-263 \text { x 125-131 } \mu \mathrm{m} \text {. }
$$

Distribución: Especie sólo conocida de España, concretamente de las provincias de Madrid, Toledo y Salamanca.

Biología: Mínguez (1981) asocia esta especie al encinar, al igual que ha ocurrido en nuestras muestras.

\author{
Ramusella (Rectoppia) strinatii s. str. \\ (Mahunka, 1980) \\ Oppia strinatii Mahunka, 1980
}

Material estudiado: Sevilla 7B (4 ej.); Sevilla 7C (1 ej.); Málaga 22B (27 ej.); Málaga 23A (7 ej.).

Dimensiones: 238-267 x 125-138 $\mu \mathrm{m}$.

Distribución: Subespecie conocida hasta ahora sólo de Marruecos.

Biología: No hay datos sobre su biología. en nuestro estudio ha aparecido asociada a alcornoque, castaño y pinsapo.

\section{Ramusella (Rectoppia) strinatii curtiramosa Subías y Rodríguez, 1987}

Material estudiado: Cádiz 10A (83 ej.); Málaga 24A (137 ej.); Granada 34A (1 ej.); Almería 41A (11 ej.).

Dimensiones: $245-288 \times 125-150 \mu \mathrm{m}$.

Distribución: Especie conocida hasta la actualidad sólo en España, concretamente en la provincia de Huesca.

Biología: La única cita conocida procedía de una muestra de hojarasca de romero en un sabinar albar. En nuestro estudio ha aparecido en hojarasca de diversas especies.

\section{Referencias}

Arillo, A., Bordel, I. \& Subías, L.S., 1988. Los oribátidos (Acari, Oribatida) de la Ciudad Universitaria de Madrid. Bol. R. Soc. Esp. Hist. Nat. (Biol.), 84: 117-125.

BECK, L. \& WoAs, S., 1991. Die Oribatiden-Arten (Acari) eines südwestdeutschen Buchenwzldes I. Carolinea, 49: 37-82. 
Fujita, M. \& Fujikawa, T., 1986. List and description of Oribatid mites in the forest litter as materials introducing soil animals into crop field of Nayoro I. Edaphologia, 35: 5-18

ItURRONDOBEitia, J.C. \& SAlOÑA, M., 1988. La familia Oppiidae (Acari, Oribatida) en Vizcaya y zonas afines. Cuad. Invest. Biol. (Bilbao), 13: 107-135.

Mínguez, M.E., 1981. Estudio taxocenótico de los Oribátidos (Acarida, Oribatida) de El Pardo. (Editorial de la Universidad Complutense de Madrid), $281 \mathrm{pp}$.

SubíAS, L.S., 1980a. Oppiidae del complejo Clavipectinata- insculpta (Acarida, Oribatida). Eos, 54: 281-313.

SubíAs, L.S., 1980b. Acaros Oribátidos de la Sierra de Cazorla (Acarida, Oribatei). Monografías I.C.O.N.A. $\mathrm{n}^{\mathrm{o}}$ 23. Viedma, M.G. (Ed). 129pp.
Subías, L.S. \& BALOGH, P., 1989. Identification keys to the genera of Oppiidae Grandjean,1951 (Acari, Oribatei). Acta Zool. Hung., 35(3/4): 355-412.

Subías, L.S. \& Rodríguez, P., 1987. Oppiidae (Acari, Oribatida) de los sabinares (Juniperus thurifera) de España I. Ramusella s. str. Hammer y Ramusella (Rectoppia) Subías. Eos, 63: 301-314.
Recibido, el 26-III-1996

Aceptado, el 2-IX-1996

Publicado, el 30-XII-1996 